\title{
Evaluation Method of Shaft Structure Stability in Coal Mine Raise Boring Engineering
}

\author{
Guo-ye JING ${ }^{1,2,3}$, Feng GAO ${ }^{1,2,3, a}$, Shou-ye CHENG ${ }^{1,2,3}$, Bo HAN ${ }^{1,2,3}$ and Junfeng LI $^{1,2,3}$ \\ ${ }^{1}$ Mine Construction Branch, China Coal Research Institute, Beijing, 100013, China \\ 2 Beijing China Coal Mine Engineering Co. Ltd, Beijing, 100013, China \\ ${ }^{3}$ National Engineering Laboratory for Deep Shaft Construction Technology in Coal Mine, Beijing, 100013, China
}

\begin{abstract}
Raise boring method is one of the key technologies for coal mine shaft construction. The typical engineering of raise boring method is two types: large diameter shafts and small diameter gas pipeline shafts. Ensuring the stability of these two types of shaft structures is a key issue that determines the quality of raise boring engineering. In order to solve this problem, a method of evaluating the stability of shaft structure constructed by raise boring method is proposed in this paper. First, for large-diameter shafts, it is clear that the stability of surrounding rock is the key factor. Based on the strength reduction method, the stability coefficient of surrounding rock $k_{0}=2.5$ is proposed. Secondly, for the small diameter gas pipe shaft, it is clear that the stability of the gas pipe under the pressure of the filling material is the key factor. The functional equations of allowable compressive stress of the pipe material, the initial deviation of the gas pipes and the ultimate pressure are established, and a method with results biased towards safety is proposed for the design and verification of gas pipes.
\end{abstract}

\section{Introduction}

In the process of coal mine construction and production, many vertical and inclined shafts that connect the upper and lower levels are required, such as transportation shafts, ventilation shafts, gas pipeline shafts, coal bunkers, coal slip holes and drain holes ${ }^{[1]}$. Due to the limitation of construction conditions or from the perspective of improving work efficiency, saving ventilation, and reducing costs, raise boring methods are often used ${ }^{[2]}$. The process flow is as follows: firstly, a small cross-section guide hole is drilled down from the upper level tunnel or chamber to connect with the lower leve ${ }^{[3]}$. When the problems of ventilation, drainage, and gangue discharge are solved, then the diameter is enlarged from bottom to top ${ }^{[4]}$.

Among a large number of shaft projects, raise boring technology is most commonly used in the construction of ventilation shafts and gas pipeline shafts ${ }^{[5]}$. Among them, the ventilation shaft is characterized by large diameter and deep depth ${ }^{[6]}$. The gas pipeline shaft is characterized by its small diameter, but the installation of gas pipelines is required ${ }^{[7]}$.

In the process of raise boring construction in coal mines, most of the safety accidents are caused by the instability of the shaft structure ${ }^{[8]}$. This paper analyzes the key problems of vertical shaft structural instability in view of two typical raise boring working conditions: the large-diameter shaft and small-diameter gas pipeline shaft. For large-diameter shafts, an engineering judgment method for surrounding rock instability based on strength reduction method is proposed. For the small diameter gas pipeline shaft, a theoretical formula for calculating the structural stability of the gas pipeline is proposed.

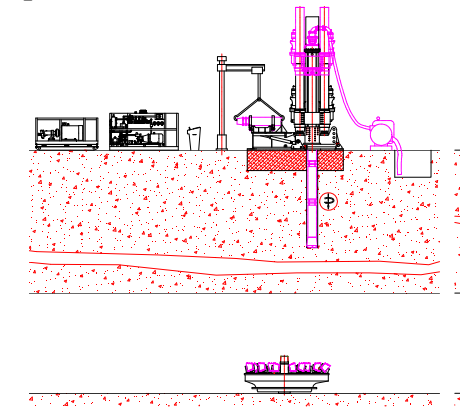

(a) Pilot hole drilling process

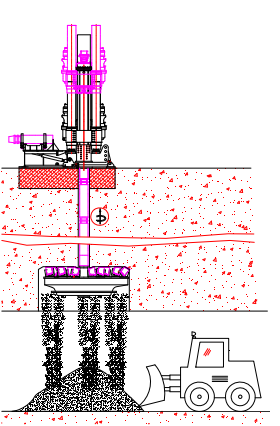

(b)Reaming process
Fig. 1 Raise boring method

\section{The structural stability of large diameter shaft}

The main influencing factor of large-diameter shafts is the instability of surrounding rock ${ }^{[9]}$. During the reaming process, the slag continuously falls from the heading face to the bottom of the shaft, and there is no working condition for personnel to enter the shaft to support the surrounding rock ${ }^{[10]}$. The surrounding rock of the shaft needs to maintain the stability of the shaft structure for several days to tens of days by virtue of its selfstabilization ability ${ }^{[11]}$. During this period, the surrounding rock is completely exposed and will be

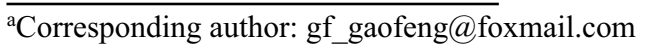


affected by ground stress, water spraying, and weathering, and the wellbore structure is very prone to instability.

In this section, the strength reduction method is used, and the goal is to determine a method for evaluating the stability of the shaft structure. Considering the state of the surrounding rock of the vertical shaft under the conditions of $400 \mathrm{~m}$ buried depth and $5 \mathrm{~m}$ diameter as the ultimate self-stability state, the safety factor $\mathrm{k} 0$ of the surrounding rock in this state is obtained by the finite difference strength reduction method. As a quantitative indicator to measure whether the surrounding rock can stabilize itself.

\subsection{Strength reduction method}

The strength reduction method is mainly to divide the rock mass strength index cohesion $c$ and internal friction angle $\varphi$ by a reduction coefficient $\mathrm{k}$ at the same time to obtain a new set of $c^{\prime}$ and $\varphi^{\prime}$ values, which are used as new material parameters for numerical calculation. When the surrounding rock just reaches the critical failure state, the corresponding $k$ value is the stability coefficient of the surrounding rock, and its calculation formula is:

$$
\begin{gathered}
c^{\prime}=c / k \\
\varphi^{\prime}=\arctan (\tan (\varphi / k))
\end{gathered}
$$

In order to avoid the blindness of the value, the numerical simulation process is first calculated according to the actual strength parameters of the rock mass, and then $k$ is uniformly increased or decreased according to the observed damage of the surrounding rock, until the deformation state of the surrounding rock approaches the critical failure In the state, a local fine value is performed to ensure the accuracy of the safety factor of the surrounding rock.

\subsection{Typical raise boring project model}

First, use Abaqus software to establish a finite element mesh, the mesh type is a four-node tetrahedral element. Then import the grid into FLAC3D for excavation simulation. The model diagram is shown in Figure 2. The size of the model is $50 \mathrm{~m} \times 50 \mathrm{~m} \times 100 \mathrm{~m}$, and the excavation area of the raise boring shaft is a cylinder with a diameter of $5 \mathrm{~m}$. The number of units in the model is 164970 and the number of nodes is 88170 . The displacement monitoring of the surrounding rock in the excavation area is shown in Figure 2. A total of 8 pairs of monitoring points are set. Based on the symmetry, the analysis can only focus on the displacement changes on one side. The raise boring area is a cylindrical area between points $\mathrm{A}$ and $\mathrm{H}$ in Figure 3, which is divided into 11 sections with a length of $6 \mathrm{~m}$.

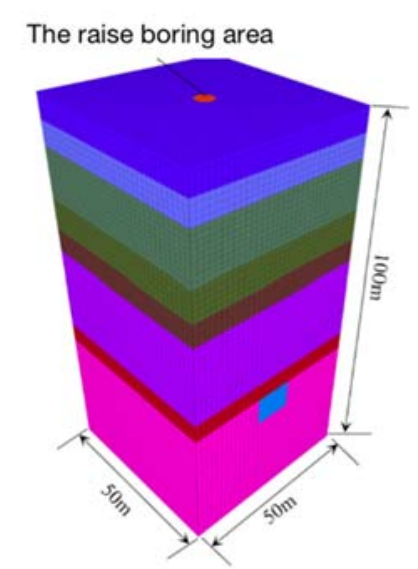

Fig. 2 Schematic diagram of model

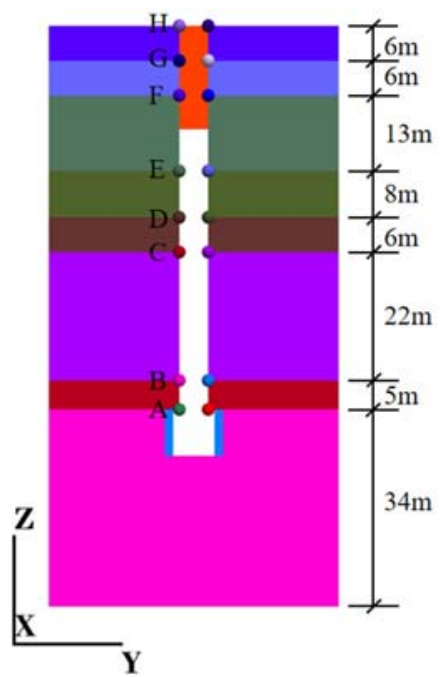

Fig 3 Schematic diagram of monitoring points

The physical and mechanical indexes of surrounding rock are selected according to the parameter combination of the most unfavorable surrounding rock stability in accordance with the "Code for Design of Railway Tunnels". The strength and modulus parameters are the lowest, and the gravity and Poisson's ratio are the highest. The rock density $\rho$ is taken as $2800 \mathrm{~kg} / \mathrm{m}^{3}$, the elastic modulus $E$ is taken as $33 \mathrm{GPa}$, the Poisson's ratio $\mu$ is taken as 0.2 , the internal friction angle $\varphi$ is taken as $60^{\circ}$, and the cohesion force $c$ is taken as $2.1 \mathrm{MPa}$.

\subsection{Numerical simulation process}

Numerical simulation can be divided into the following 5 steps:

Step 1 . Set the boundary conditions, set the normal displacement constraints along the boundary surface around the model, and set the displacement constraints in the three directions at the bottom to perform initial ground stress balance.

Step 2. After the ground stress is balanced, apply a compressive stress perpendicular to the boundary on the top boundary of the model with a size of $400 \times 25000=10 \mathrm{MPa}$ to simulate the stress state of the 
surrounding rock at a buried depth of $400 \mathrm{~m}$.

Step 3. Excavate the soil in the "roadway" area at the seventieth point of the raise well (zone cmodel assign null), and perform the ground stress balance again.

Step 4. Perform 11 excavations along the excavation sequence of $\mathrm{A} \rightarrow \mathrm{H}$ in the excavation section of raise drilling.

Step 5. Control other parameters of the surrounding rock unchanged, change the values of $\mathrm{c}$ and $\varphi$ according to the strength reduction coefficient, and repeat the process from step 1 to step 4 .

\subsection{Calculation results and analysis}

The strength reduction method is used to calculate the deformation state of the surrounding rock under different reduction coefficients, and the displacement values of the key points on the shaft wall under each strength reduction coefficient are calculated.

Under the conditions of burial depth of $400 \mathrm{~m}$, diameter of $5 \mathrm{~m}$ and Class I surrounding rock, the relationship between the strength reduction coefficient and the key point displacement (y direction) after excavation is shown in Figure 34 It can be seen from Fig. 3 that the displacement of key points basically shows a trend of simultaneous growth. When $k<2.5$, the displacement value increases slowly, indicating that the surrounding rock is in the elastic deformation stage. When $k>2.5$, the displacement value increases rapidly, indicating that some surrounding rock units have entered a stage of infinite development of shaping and flow.

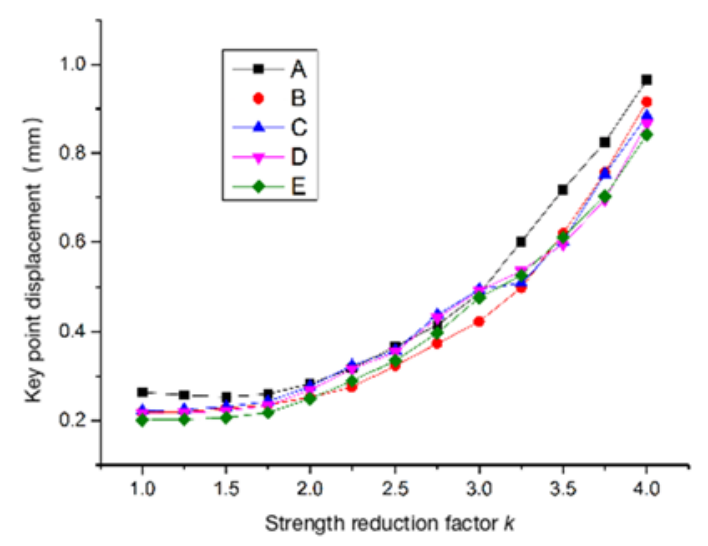

Fig 4 Relationship between strength reduction coefficient and key point displacement value

The development of the surrounding rock plastic zone is shown in Figure 5. Take the horizontal section through point $B$ under each strength reduction coefficient when the excavation is $48 \mathrm{~m}$ respectively for comparison. It can be seen from Figure 4 that when $k<2.75$, the area of the plastic zone increases sharply with the increase of $k$; when $k>2.75$, the area of the plastic zone does not increase with the increase of $k$. This is due to the boundary effect and the model selection The scope is limited. When $k<2.75$, the shape of the plastic zone is approximately circular; when $k>2.75$, the shape of the plastic zone is similar to a "cross" shape due to the boundary effect.

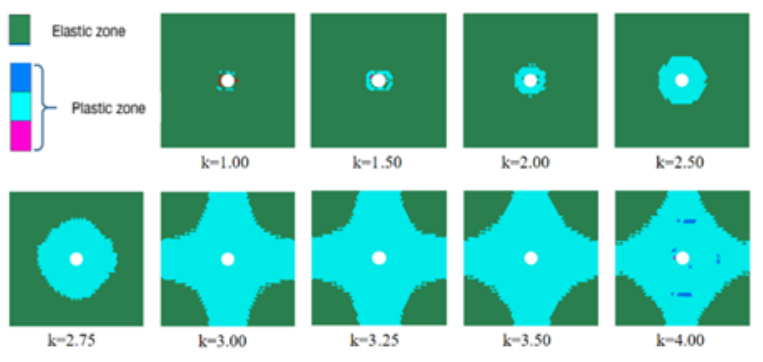

Fig 5 Development of surrounding rock plastic zone

Using the method of color extraction, the area of the plastic zone in each graph is obtained, and then the area of the plastic zone (unit: $\mathrm{m}^{2}$ ) is obtained through proportional conversion, as shown in Figure 6. It can be seen from Figure 6 that when $k$ is less than 2.5, the area of the plastic zone increases approximately linearly. When $k$ is greater than 2.5 , the area of the plastic zone increases exponentially with the increase of $k$.

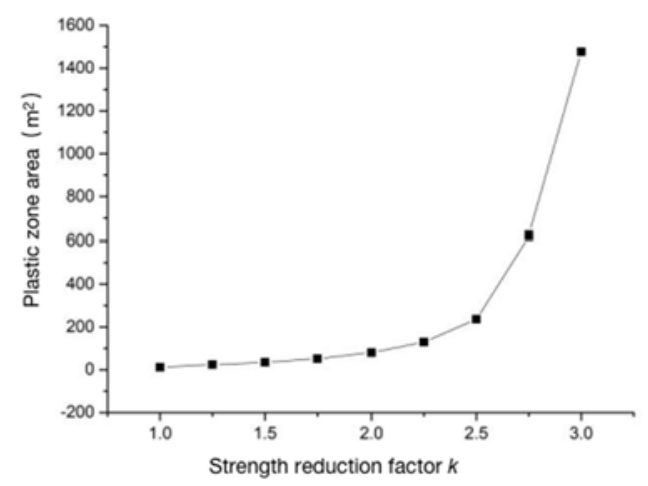

Fig 6 The relationship between plastic zone area and strength reduction coefficient

In summary, it is assumed that 2.5 is the stability factor $k_{0}$ of the surrounding rock, which means that the strength of the surrounding rock can withstand the secondary stress damage and remain stable.

When the surrounding rock stability coefficient is greater than $k_{0}$, the surrounding rock can stabilize itself. When it is less than $k_{0}$, load-bearing support and surrounding rock shall be applied to bear secondary stress failure.

\section{The structural stability of gas pipeline shaft}

Gas pipeline shaft is another common raise boring project. The main construction process of the gas pipeline shaft is divided into four steps: pilot hole drilling, reaming, placing the gas pipeline and filling behind the wall ${ }^{[12]}$. The gas pipeline shaft is characterized by a small diameter, generally no more than $3.5 \mathrm{~m}$, so the surrounding rock has good stability and high safety in the reaming stage ${ }^{[13]}$. However, compared with conventional raise boring projects, gas pipeline shafts require an additional backfilling operation. 
Place the gas pipeline first, and then pour the grouting material between the pipeline and the surrounding rock.

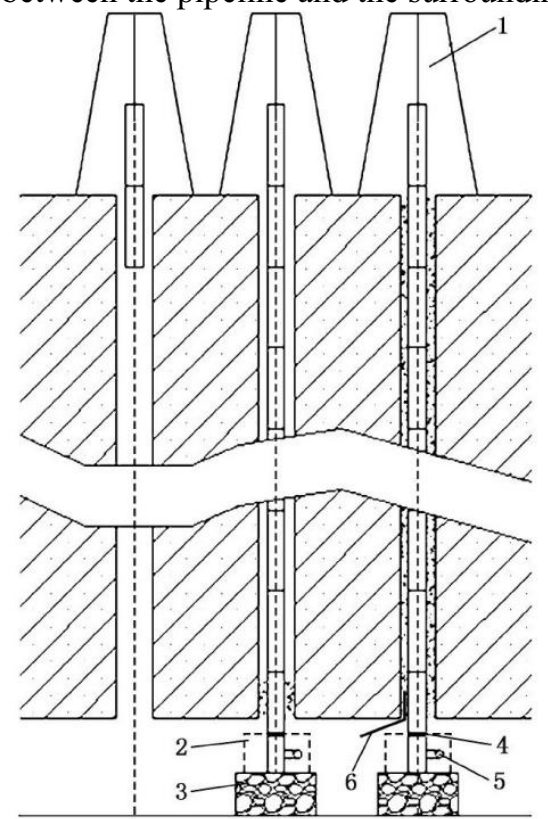

Fig 7 Gas pipeline installation and filling behind the wall

1-Lifting tower; 2-Fixing and supporting device; 3-Concrete supporting pier; 4-Bearing end; 5-Drainage valve in pipe; 6Drainage pipeline

The main factor for the structural failure of the gas pipeline shaft is the excessive pressure generated by the grouting material behind the wall on the gas pipeline, causing the pipeline to be crushed. During the filling process behind gas pipes, the grouting material behind the wall generates pressure on the body of the gas pipe, and the pressure increases with the increase in depth, leading to the risk of collapse of some pipe sections due to external pressure. If the bending rigidity of the pipe body material is insufficient, it may collapse under a stress far below the elastic limit of the material, which seriously restricts the safety of the gas pipes.

\subsection{Buckling of Gas Pipe Section Ring}

Before discussing the buckling of the entire pipe section under pressure, first analyze the buckling conditions of the gas pipe section ring. The ring will buckle into an ellipse under the action of external pressure, as shown by the dotted line.

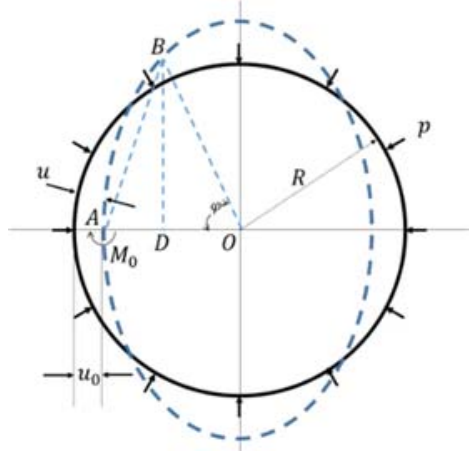

Fig 8 Gas Buckling of Gas Pipe Section Ringpipeline installation and filling behind the wall $p$ : External pressure within unit length of center line

$R$ : Radial displacement of the ring centerline

$u$ : Buckling is the radial displacement

$u_{0}$ : Radial displacement of section A

$M_{0}$ : Bending moment at section A

The bending moment at any section $\mathrm{B}$ of the buckling ring can be expressed as:

In the triangle $\mathrm{AOB}$ :

$$
M=M_{0}+p \overline{A B} \cdot \overline{A D}-\frac{p}{2} \overline{A B}^{2}
$$

$$
\overline{O B}^{2}=\overline{A B}^{2}+\overline{A O}^{2}-\overline{A O} \cdot \overline{A D}
$$

which is:

$$
\frac{1}{2} \overline{A B}^{2}-\overline{A O} \cdot \overline{A D}=\frac{1}{2}\left[(R-u)^{2}-\left(R-u_{0}\right)^{2}\right]
$$

Relative to $R, u$ has a small value, so ignoring terms $u^{2}$ and $u_{0}^{2}$, we get:

$$
\frac{1}{2} \overline{A B}^{2}-\overline{A O} \cdot \overline{A D}=R\left(u-u_{0}\right)
$$

Combined with the general equation of the deflection curve, we can get:

$$
\begin{aligned}
& \frac{d^{2} u}{d \varphi^{2}}+u\left(1+\frac{p R^{3}}{E I}\right)=\frac{-M_{0} R^{2}+p R^{3} u_{0}}{E I} \\
& \frac{d^{2} u}{d \varphi^{2}}+u=-\frac{R^{2}}{E I}\left[M_{0}-p R\left(u-u_{0}\right)\right]
\end{aligned}
$$

Combine similar items, get:

$$
\frac{d^{2} u}{d \varphi^{2}}+u\left(1+\frac{p R^{3}}{E I}\right)=\frac{-M_{0} R^{2}+p R^{3} u_{0}}{E I}
$$

The general solution of this equation is:

$$
u=C_{1} \sin k \varphi+C_{2} \cos k \varphi+\frac{-M_{0} R^{2}+p R^{3} u_{0}}{E I+p R^{3}}
$$

Where $C_{1}$ and $C_{2}$ are constants determined by the conditions at the section $A$ and $F$ of the buckling ring,

$$
k^{2}=1+\frac{p R^{3}}{E I}
$$

From the symmetry:

$$
\left(\frac{d u}{d \varphi}\right)_{\varphi=0}=0 ; \quad\left(\frac{d u}{d \varphi}\right)_{\varphi=\pi / 2}=0
$$

From the first condition, $C_{1}=0$ is obtained, and from the second condition:

$$
\sin \frac{k \pi}{2}=0
$$

The smallest root of this equation is:

$$
\frac{k \pi}{2}=\pi
$$

Substituting into equation (11), the value of critical stress is:

$$
p_{c r}=\frac{3 E I}{R^{3}}
$$

The other roots of formula (a), such as $k \pi / 2=2 \pi$, $k \pi / 2=3 \pi$, etc., correspond to the larger wave numbers in the buckling ring, and the remaining larger values of pressure $p$ can be obtained. These values only have an effect when the pipe section is short, but the actual gas pipe In the project, the length of the gas pipe is much larger than its diameter, so the other roots of formula (a) will not be discussed in depth.

\subsection{Buckling of long round gas pipeline under ideal conditionsg}

Based on the above discussion of the buckling of the ring of the gas pipe section, it is extended to the situation that the long round gas pipe bears uniform external pressure under ideal conditions. Analyze the micro-ring cut from the gas tube by two sections separated by unit length. The section moment of inertia of this ring is: 


$$
I=\frac{1 \cdot h^{3}}{12}
$$

In the formula, it is used to indicate the thickness of the gas pipeline. Since the cross-section of the ring will not be distorted when bent, it is necessary to use Formula (15) instead of $E$.

$$
\frac{E}{1-u^{2}}
$$

The equation (13) used to calculate the critical pressure becomes equation (16)

$$
p_{c r}=\frac{E h^{3}}{4\left(1-\mu^{2}\right) R^{3}}
$$

As long as the corresponding compressive stress in the gas pipe is less than the proportional limit of the pipe wall material, the above formula can be used. When the elastic limit is exceeded, the true critical pressure will be less than the result obtained by equation (16). The wall of gas pipe is generally made of steel with obvious yield point. The following equation can be used:

$$
p_{c r}=\frac{h}{R} \cdot \frac{\sigma_{s}}{1+4 \frac{\sigma_{S} R^{2}}{E h^{2}}}
$$

In the formula, $\sigma_{s}$ represents the yield point when the pipe wall material is compressed. As the thickness decreases, the critical pressure approaches the limit value $E h^{3} / 4 R^{3}$, which is slightly smaller than that shown in equation (16), and in all cases its value is less than $h \sigma_{s} / R$, that is, less than the pressure corresponding to the yield point stress .

In the area exceeding the elastic limit, it can be treated as a column in the mechanics of materials, and $E_{t}$ is used instead of $E$ in equation (16). In this way, the relationship between $p_{c r}$ and $h / R$ is established in the plastic range. In actual engineering, the gas pipe material should be prevented from entering the plastic zone, so the situation of exceeding the elastic limit will not be discussed in detail here.

\subsection{Buckling of gas pipe with initial defects}

The failure of the gas tube under uniform external pressure is closely related to its own defects in the process of processing, construction and installation. The most important defect that affects its crushing failure is the initial ellipticity of the section of the gas tube after it is installed. In any type of gas pipe, the initial ellipticity limit value of the entire pipe section can be effectively measured on the project site. Therefore, the establishment of a formula to clearly reflect the relationship between the initial ellipticity of the gas pipeline and the collapse of the pipe section has important engineering practical significance.

Assuming that the initial deviation of the gas tube shape as shown by the dotted line in Figure 8 relative to the complete circle can be expressed by the following formula:

$$
u_{1}=u_{0} \cos 2 \varphi
$$

In the formula, $u_{0}$ is the maximum initial radial deviation, and its value is much smaller than $R$, and $\varphi$ is the central angle as shown in Figure 9. The initial shape of the gas tube is indicated by the solid line in the figure.

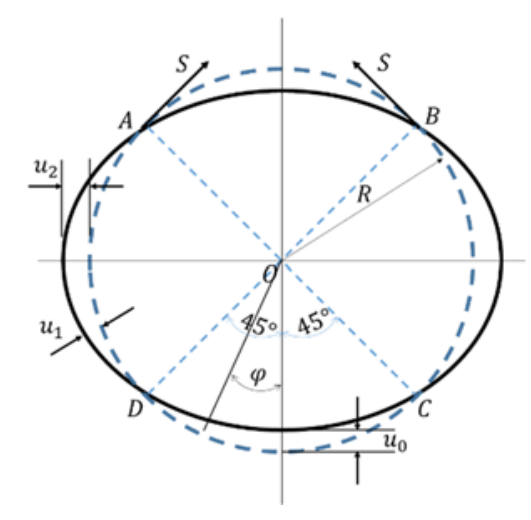

Fig 9 Buckling of gas pipe with initial defects

When there is an external pressure $p$ acting on the non-circular gas tube, the gas tube will become flatter. Let $u_{2}$ represent the radial displacement corresponding to the flattening of the gas tube, and carry out the force analysis on the unit ring with unit width at this position, and we can get:

$$
\frac{d^{2} u_{2}}{d \varphi^{2}}+\mu_{2}=-\frac{1}{D} M R^{2}
$$

Where:

$$
D=\frac{E h^{3}}{12\left(1-u^{2}\right)}
$$

$D$ is the bending stiffness of the unit ring. Regarding the bending moment $M$ generated by the pressure $p$, it can be clearly found that in the $\mathrm{AB}$ and $\mathrm{CD}$ parts of the unit ring, the curvature is reduced, so in these areas $M$ is positive, and the bending moment is negative in the rest of the ring. At points $\mathrm{A}, \mathrm{B}, \mathrm{C}$, and $\mathrm{D}$, the bending moment is zero. The interaction between the parts of the unit ring can be represented by the force $S$ tangent to the dashed line representing the ideal shape of the gas pipe (the effect of the force $S$ on the part $\mathrm{AB}$ of the ring is shown in Fig. 8). The circle can be regarded as a curve tensioned by the uniform external pressure $p$. The pressure along this curve remains constant, and its magnitude is equal to $S=p R$. Therefore, the bending moment at any section can be obtained by multiplying $S$ with the total radial deviation $u_{1}+u_{2}$ at that section. which is:

$$
\begin{aligned}
& \qquad M=p R\left(u_{2}+u_{0} \cos 2 \varphi\right) \\
& \text { And equation (19) becomes: } \\
& \frac{d^{2} u_{2}}{d \varphi^{2}}+\mu_{2}=-\frac{1}{D} p R^{3}\left(u_{2}+u_{0} \cos 2 \varphi\right)
\end{aligned}
$$

or

$$
\frac{d^{2} u_{2}}{d \varphi^{2}}+\mu_{2}\left(1+\frac{p R^{3}}{D}\right)=-\frac{1}{D} p R^{3} \cos 2 \varphi
$$

The solution of the equation satisfying the continuous conditions at $\mathrm{A}, \mathrm{B}, \mathrm{C}$ and $\mathrm{D}$ is:

$$
u_{2}=\frac{u_{0} p}{p_{c r}-p} \cos 2 \varphi
$$

Where $p_{c r}$ is obtained from equation (16). It can be seen that the displacement $\mu_{2}$ and its second derivative are zero at points $\mathrm{A}, \mathrm{B}, \mathrm{C}$ and $\mathrm{D}$. Therefore, the bending moment at these points is zero, which is consistent with the assumption put forward in the previous question. The maximum bending moment occurs at $\varphi=0$ and $\varphi=$ $\pi$, here:

$$
M_{\max }=p R\left(u_{0}+\frac{u_{0} p}{p_{c r}-p}\right)=\frac{p u_{0} R}{1-\frac{p}{p_{c r}}}
$$


It can be seen from the above formula that when the value of $p / p_{c r}$ is very small, the change in the ellipticity of the gas tube caused by the pressure $p$ can be ignored, and the maximum bending moment can be obtained by multiplying the pressure $S=p R$ and the initial deviation $u_{0}$. If the $p / p_{c r}$ is larger, the change of the initial ellipticity must be considered, and equation (25) must be used when calculating $\mathrm{M}$.

The maximum compressive stress can now be obtained by adding the maximum compressive stress generated by the bending moment $M_{\max }$ and the stress generated by the pressure $p R$. Therefore,

$$
|\sigma|_{\max }=\frac{p R}{h}+\frac{6 u_{0} p R}{h^{2}} \cdot \frac{1}{1-\frac{p}{p_{c r}}}
$$

With the aid of this equation, a method for the design and calibration of gas pipes can be developed.

The limit value of the pressure $p$ is the pressure value $p_{s}$ at which the material begins to yield. To express this value, replace $\sigma_{\max }$ with $\sigma_{s}$ in equation (26), we get:

$$
\sigma_{s}=\frac{p_{s} R}{h}+\frac{6 u_{0} p_{s} R}{h^{2}} \cdot \frac{1}{1-\frac{p_{s}}{p_{c r}}}
$$

In actual engineering, the allowable compressive stress $\sigma_{s}$ of the pipe wall material and the initial deviation $\mu_{0}$ of the gas pipeline can be obtained, and the value of the ultimate pressure $p_{s}$ can be calculated from the above formula. Make:

$$
m=\frac{R}{h}, n=\frac{u_{0}}{R}
$$
(29):

Equation (27) used to calculate $\mathrm{P}$ becomes equation

$$
p_{s}^{2}-\left[\frac{p_{s}}{m}+(1+6 m n) p_{c r}\right] p_{s}+\frac{p_{s} p_{c r}}{m}=0
$$

From this equation, the curves of average compressive stress $p_{s}$ and $R / h$ can be obtained. For $u_{0} /_{R}$ and $\sigma_{s}$ under different values, the average compressive stress $p_{s}$ is a function of $R / h$. In engineering practice, the average compressive stress $p_{s}$ can be the hydrostatic pressure of the filling material at a specified depth. On the basis of this curve, combined with a reasonable safety factor, the gas pipe wall thickness that meets the conditions can be quickly obtained.

This method fully considers the initial defects of the gas tube, and the pressure $p_{s}$ in the equation is always less than the actual pressure when the gas tube is completely collapsed. Therefore, if the $p_{s}$ in this theory is used as the ultimate pressure, the result is always safe. Therefore, this method is suitable for actual gas pipeline shaft engineering.

\section{Conclusion}

In this paper, the in-depth analysis of the factors that affect the structural stability when the raise drilling method is used to construct a shaft in the coal mine field. For large diameter shafts, the key factor is the stability of the surrounding rock during the reaming process. For small diameter gas pipeline shafts, the key factor is the stability of the gas pipeline under the pressure of the filling material.

For large-diameter shafts, this paper uses the strength reduction method to determine the strength reduction coefficient $k_{0}=2.5$ of the surrounding rock's ultimate selfstability state through numerical simulation, and uses it as the stability coefficient of the surrounding rock. When the surrounding rock stability coefficient is greater than $k_{0}$, the surrounding rock can stabilize itself. When it is less than $k_{0}$, load-bearing support and surrounding rock shall be applied to bear secondary stress failure.

For gas pipeline shafts, this paper establishes the allowable compressive stress $\sigma_{s}$ of the pipe material, the initial deviation $\mu_{0}$ of the gas pipes and the ultimate pressure $p_{s}$ through theoretical derivation. A method with results biased towards safety is proposed for the design and verification of gas pipes.

\section{Acknowledgement}

This paper was funded by the Innovation and Entrepreneurship Fund of Tiandi Technology Co., Ltd. The project number is 2019-MS-006

\section{References}

1. LIU Zhi-qiang. Research on process and key technologies of raise boring [J]. Coal Science and Technology, 47(05):12-21(2019).

2. JING Guoye. Construction technology of reaming once with raiseborer in large diameter staple shaft [J]. China Mining Magazine, 27(01):123$126+136(2018)$.

3. Gao Feng, Liu Zhiqiang, Zhou Huaqun, Cheng Shouye. Analysis of longitudinal vibration of raiseboring machine under the reaming condition [J]. China Coal, 43(12):96-101(2017).

4. CHENG Shou-ye. Study and Application of Raiseboring Method for Deep Vertical Shaft of Railway Tunnel Construction: a Case Study of Dangjinshan Tunnel [J]. Tunnel Construction, 38(09):15601565(2018).

5. TANG Zheng, GAO Feng, CHENG Shou-ye, LONG Zhi-yang, LIU Zhi-qiang. Influence of Mudstone Formation Softening on Shaft Wall Stability of Raise Boring Enlarging [J]. Coal Engineering, 51(12):24-28(2019).

6. JING Guo-ye, HAN Bo, LIU Zhi-qiang. Research onsinking technology of full-section shaft boring machine [J]. Coal Engineering, 52(10):29-33(2020).

7. LIU Zhi-qiang. Large diameter raise boring machine and raise boring technology $[\mathrm{J}]$. Coal Science and Technology, (11):1-3(2008).

8. JING Guo-ye. Research on Key Construction Technology of Large Diameter Raise Boring Through Special Stratum [J]. Coal Engineering, 51(05):68-72(2019).

9. CHENG Shouye, MA Yibo, YU Jianshui. Research of raise boring technology on constructing shaft in 
gold mine and engineering application [J]. China Mining Magazine, 28(04):115-119(2019).

10. GAO Feng, JING Guoye. Raise Boring Technology of Large Diameter Blind Shaft in Phosphorus Mine [J]. Mine Construction Technology, 39(01):610(2018).

11. JING Guoye. New Technology of Raisebore in Large Diameter and Deep Shaft Construction [J]. Coal Technology,33(08):66-68(2014).

12. Cheng Shouye. Overview on Development of Raise Boring Technology in China [J]. Mine Construction Technology, 38(05):14-17+21(2017).

13. Cheng Shouye. Research of raise boring technology on constructing nearly $300 \mathrm{~m}$ deep shaft underground in iron mine [J]. China Mining Magazine, 23(07):96-99(2014). 